\title{
Discovery of small-molecule interleukin-2 inhibitors from a DNA-encoded chemical library
}

\section{Doctoral Thesis}

\section{Author(s):}

Leimbacher, Markus

Publication date:

2012

Permanent link:

https://doi.org/10.3929/ethz-a-007328080

Rights / license:

In Copyright - Non-Commercial Use Permitted 
DISS. ETH NO. 20447

\section{DISCOVERY OF SMALL-MOLECULE INTERLEUKIN-2 INHIBITORS FROM A DNA-ENCODED CHEMICAL LIBRARY}

A dissertation submitted to

ETH Zurich

For the degree of

Doctor of Sciences

presented by

Markus Leimbacher

Eidg. dipl. Apotheker, ETH Zürich

Born 27 May 1981

Citizen of Winterthur $(\mathrm{ZH})$

Accepted on the recommendation of

Prof. Dr. Dario Neri, examiner

Prof. Dr. Karl-Heinz Altmann, co-examiner 


\section{ABSTRACT}

The identification of novel small-molecule ligands against medically relevant proteins is a central challenge in pharmaceutical sciences. However, the discovery of binding compounds by conventional high-throughput screening (HTS) campaigns is highly demanding in terms of time and resources. DNA-encoded chemical libraries (DELs) represent a selection-based ligand-identification methodology, which addresses the need for substantially more efficient technologies than HTS for hit discovery programs.

DELs consist of small organic molecules, which are individually coupled to DNA tags serving as PCR-amplifiable identification bar codes. DNA-encoding facilitates a selection-based isolation of binding molecules from large compound repertoires by affinity capture experiments against immobilized proteins of interest, in analogy to established selection methodologies like antibody phage display. This thesis describes the rational design and synthesis of a high-quality 30,000-membered DEL (DEL30000 library), aiming at overcoming current methodological limitations in the field.

The DEL30000 library was used to systematically investigate affinity selection protocols using the tumor-associated antigen human carbonic anhydrase IX (CA IX) as model target. Selection results suggest that experimental conditions in affinity selections critically influence selection results and thus the choice of compounds to be resynthesized for hit validation. The investigation of affinity selection protocols led to the identification of two CA IX inhibitors based on phenyl sulfonamide building blocks with inhibitory constants of $74 \pm 11 \mathrm{nM}$ (compound $\mathrm{A}_{6} \mathrm{~B}_{170}$ ) and of $76 \pm 13 \mathrm{nM}$ (compound $\mathrm{A}_{69} \mathrm{~B}_{151}$ ). Notably, fully assembled hit compounds exhibited up to 7-fold improved inhibitory constants compared to primary phenyl sulfonamide building blocks $(560 \pm$ $130 \mathrm{nM}$ for compound $\mathrm{B}_{151}$ and $230 \pm 14 \mathrm{nM}$ for compound $\mathrm{B}_{170}$ ).

A large-scale affinity screening campaign against 28 proteins of pharmaceutical interest led to the identification of a compound series of 2-methyl-1H-indoles, which was exclusively enriched in an affinity selection against the pro-inflammatory cytokine human interleukin-2 (IL-2). The 
most potent compound $\left(\mathrm{A}_{17} \mathrm{~B}_{284}\right)$ exhibited a dissociation constant of $2.5 \pm 0.3 \mu \mathrm{M}$ against IL-2 $\left(\right.$ Proleukin $^{\circledR}$ ) in fluorescence polarization assays and was able to completely inhibit IL-2-mediated T-cell proliferation with an $\mathrm{IC}_{50}$ of $32 \pm 3 \mu \mathrm{M}$. The target specificity of isolated IL-2 inhibitors was confirmed in different in vitro assays. Both affinity selection readouts and molecular docking suggest that the 2-methyl- $1 H$-indole moiety represents the structural key determinant for IL-2 recognition. 


\section{ZUSAMMENFASSUNG}

Die meisten pharmazeutischen Wirkstoffe üben therapeutische (und unerwünschte) Effekte durch Interaktionen mit einem oder mehreren spezifischen Proteinen in einem Organismus hervor. Die Entdeckung neuer, organischer Verbindungen, die mit medizinsch relevanten Proteinen in Wechselwirkung treten können, ist eine Herausforderung in den pharmazeutischen Wissenschaften. Die gegenwärtig geläufigste Strategie zur Identifikation neuer Wirkstoffe (High-throughput screening) ist allerdings zeitaufwändig und sehr kostspielig. DNA-codierte chemische Bibliotheken stellen eine neuartige Methode dar, die es in effizienter Weise ermöglicht, sehr grosse Substanzbibliotheken simultan auf Bindungsaffinitäten gegen ein Zielprotein der Wahl zu testen. DNA-codierte chemische Bibliotheken umfassen eine sehr grosse Anzahl verschiedener organischer Verbindungen, die individuell an spezifische DNA-Sequenzen gekoppelt sind. Dies ermöglicht es, die gesamte Bibliothek in Selektionsexperimenen einem immobilisierten Protein zu exponieren, und angereicherte Verbindungen durch Entschlüsselung ihres spezifisch DNA-codes zu identifizieren.

Die vorliegende Dissertation beschreibt die Synthese einer DNA-codierten chemischen Bibliothek, die 30’000 verschiedene Verbindungen umfasst und strenge Qualitätsanforderungen erfüllt. Die Bibliothek wurde systematisch in Affinitätsselektionen gegen die menschliche Carboanhydrase IX (CA IX) - ein Membran-gebundenes Enzym, welches in vielen Tumortypen stark überexprimiert ist - getestet, um den Einfluss unterschiedlicher experimenteller Bedingungen in Affinitätsselektionen auf Selektionsergebnisse zu prüfen. Es stellte sich heraus, dass bestimmte experimentelle Faktoren einen entscheidenden Einfluss auf Selektionsresultate ausüben können und damit auf die Wahl, welche Verbindungen detektiert und für Validierungstests resynthetisiert werden. Diese Experimentreihe gegen CA IX führte zur Identifikation mehrerer, starker Inhibitoren (Phenylsulfonamid-Derivate), die die CA IX Aktivität mit nanomolaren Inhibitionskonstanten hemmten (z.B.: Verbindung $\mathrm{A}_{6} \mathrm{~B}_{170}$ mit $74 \pm 11 \mathrm{nM}$ und Verbindung $\mathrm{A}_{69} \mathrm{~B}_{151}$ mit $76 \pm 13 \mathrm{nM}$ ). 
Daraufhin wurde die DNA-codierte chemische Bibliothek in Selektionsexperimenten gegen weitere 28 Proteine von pharmazeutischem Interesse geprüft, wobei eine Reihe von 2-Methyl-1HIndol-Derivaten gegen das entzündungsfördernde Zytokin Interleukin-2 (IL-2) stark und selektiv angereichert war. Die beste Verbindung aus dieser Reihe $\left(\mathrm{A}_{17} \mathrm{~B}_{284}\right)$ band IL-2 mit einer Dissoziationskonstante von $2.5 \pm 0.3 \mu \mathrm{M}$ und hemmte die IL-2-abhängige Proliferation von T-Zellen vollständig mit einer mittleren inhibitorischen Konzentration von $32 \pm 3 \mu \mathrm{M}$. Andere Testverbindungen zeigten keine Bindungsaktivität gegen IL-2, während die Verbindung $\mathrm{A}_{17} \mathrm{~B}_{284}$ keine messbare Bindungsaktivität gegen Streptavidin aufwies. Ein chemoinformatisches Verfahren zeigte zudem auf, dass die 2-Methyl-1H-Indol-Substruktur der Verbindung $\mathrm{A}_{17} \mathrm{~B}_{284}$ entscheidund für die Bindung an IL-2 ist, was im Einklang mit den Selektionsergebnissen stand. 